\title{
Gene silencing of claudin-6 enhances cell proliferation and migration accompanied with increased MMP-2 activity via p38 MAPK signaling pathway in human breast epithelium cell line HBL-100
}

\author{
YUE REN, QIONG WU, YAFANG LIU, XIAOMING XU and CHENGSHI QUAN \\ Key Laboratory of Pathobiology, Ministry of Education, Bethune Medical College, \\ Jilin University, Changchun, Jilin 130021, P.R. China
}

Received May 2, 2013; Accepted August 12, 2013

DOI: $10.3892 / \mathrm{mmr} .2013 .1675$

\begin{abstract}
Disruption or loss of tight junction structure and function is associated with tumor growth, invasion and metastasis in tumors of human epithelial origin. Since claudin is the most important backbone protein of tight junctions, the downregulation or loss of claudin expression is hypothesized to be important for tumor development and metastasis. In the current study, RNA interference (RNAi) was used to knock down the expression of claudin- 6 to investigate the effect of claudin- 6 downregulation on the malignant phenotype in the human breast epithelium cell line HBL-100. The junctional function was investigated by measuring the transepithelial electrical resistance across the confluent epithelial cell layer. Manual cell counting and wound healing assays were performed to examine cell proliferation and migration. Changes in matrix metalloproteinase-2 (MMP-2) expression and activity were examined by reverse transcription polymerase chain reaction (RT-PCR) and gelatin zymography. The expression of p38 mitogen-activated protein kinases (MAPKs) and phosphorylated p38 MAPK were measured by western blot analysis. Claudin-6 knockdown resulted in significantly lower transepithelial electrical resistance $(\mathrm{P}<0.001)$, higher growth rate $(\mathrm{P}<0.001)$ and migratory ability $(\mathrm{P}<0.001)$ accompanied with an increased MMP-2 expression and activity $(\mathrm{P}<0.001)$. Furthermore, a decreased expression of phosphorylated p38 MAPK $(\mathrm{P}<0.001)$ was detected in HBL-100 cells. These observations support the hypothesis that a decreased expression of claudin- 6 contributes to the malignant progression of human breast cancer.
\end{abstract}

Correspondence to: Professor Chengshi Quan, Key Laboratory of Pathobiology, Ministry of Education, Bethune Medical College, Jilin University, 126 Xinmin Avenue, Changchun, Jilin 130021, P.R. China

E-mail: quancs@jlu.edu.cn

Key words: claudin-6, tight junction, breast cancer, matrix metalloproteinase-2, p38 mitogen-activated protein kinase

\section{Introduction}

Tight junctions are intercellular structures, located at the top of junctional complexes, which are critical in maintaining the fence, barrier and signal transduction functions in epithelial and endothelial cells (1-3). Tight junctions are composed of three types of integral membrane proteins: occludins, claudins and junction adhesion molecules and also contain peripheral plasmosins, including ZO-1, -2 and -3 (4). Claudins, which contain at least 27 members, have been shown to be the most important backbone protein of tight junctions (5). Claudin-6 is a protein in the claudin family with a molecular weight of $\sim 23 \mathrm{kDa}$ and whose gene is located at chromosome 16p13.3.

There is increasing evidence indicating that tight junctions are involved in cell migration, proliferation, differentiation and apoptosis. Disruption or loss of tight junction structure and function has been reported in tumors of human epithelial origin and it has been shown that these changes are associated with variations in tumor growth, invasion and metastasis (6-8). Thus, the loss or downregulated expression of claudin and other tight junction proteins is hypothesized to be important steps for tumor development and metastasis (9).

Previously, claudin-6 was identified as a potential breast cancer suppressor gene, which may contribute to a breast cancer resistant phenotype observed in Copenhagen rats (10). In addition, overexpression of claudin- 6 may abate the malignant phenotype of the human breast cancer cell line MCF-7 (11), indicating that claudin-6 may play a role in suppressing the development of breast cancer.

In the current study, RNA interference (RNAi) was used to knock down the expression of claudin- 6 in the human breast epithelium cell line HBL-100. By examining the junctional function, proliferation and migration, the aim was to determine whether the loss of claudin-6 may have any effects on HBL-100 cells. The results clearly showed that the downregulation of claudin-6 in HBL-100 cells leads to a higher growth rate and migratory ability, accompanied with an increased MMP-2 expression and activity, which may be mediated via the p38 MAPK pathway. These observations support the hypothesis that a decreased expression of claudin- 6 contributes to the malignant progression of breast cancer. 


\section{Materials and methods}

Cell line. Human breast epithelial cell line HBL-100 was obtained from the American Type Culture Collection and maintained in DMEM medium (Gibco-BRL, Carlsbad, CA, USA) supplemented with $10 \%$ fetal bovine serum (Gibco) at $37^{\circ} \mathrm{C}$ in $5 \% \mathrm{CO}_{2}$.

Short hairpin RNA (shRNA) transfection. Four shRNAs were designed, based on the claudin-6 mRNA sequence NM_021195 and were constructed into the vector pGCsilencer ${ }^{\mathrm{TM}} \mathrm{U} 6 / \mathrm{Neo} /$ GFP (GeneChem, Shanghai, China). The shRNAs were transfected into cells using SuperFect ${ }^{\circledR}$ transfection reagent (Qiagen, Germantown, MD, USA). Transfected cells were treated with $400 \mu \mathrm{g} / \mathrm{ml} \mathrm{G} 418$ (Sigma-Aldrich, St. Louis, MO, USA) for 10 days. Populations of knockdown cells were pooled following 10 days G418 selection. A negative control cell line was generated by transfecting cells with the vector constructed by targeting a sequence that did not yield any appreciable knockdown of the protein production. shRNA sequences were as follows: i) caGTCAAGCTATGGAACTAATTTCAAGAG AATTAGTTCCATCATAGCTTGACtg; ii) aaCTGAGCC AAGGTGTTGACTTTCAAGAGAAGTCAACACCTTGGC TCAGtt; iii) gcCAGATGCAGTGCAAGGTGTTTCAAGAG AACACCTTGCAACTGCATCTGgc and iv) caGTG CAAGGTGTACGACTCATTCAAGAGATGAGTCGTACAC CTTGCACtg.

Reverse transcription-polymerase chain reaction (RT-PCR). Total RNA was extracted using TRIzol reagent (Invitrogen Life Technologies, Carlsbad, CA, USA) and reverse-transcribed with M-MLV reverse transcriptase (Takara Bio Inc., Shiga, Japan). PCR was performed to analyze claudin-6 mRNA expression (Applied Biosystems, Carlsbad, CA, USA). Forward and reverse primers of claudin- 6 used were: 5'-CAC TGCCACTTCTGGATGG-3' and 5'-CAGTGCAGCTCCTT CAACCT-3'.

Measurement of transepithelial electrical resistance (TER). Monolayers of cells were grown in filters (Millicell culture plate inserts; Millipore, Billerica, MA, USA). The TER was measured when the cells had grown to full confluency, using a voltmeter (Millicell-ERS). TER $\left(\Omega \times \mathrm{cm}^{2}\right)=$ (total resistance - blank resistance) $(\Omega) \times$ area $\left(\mathrm{cm}^{2}\right)$. Three replicates each of three independent experiments were performed.

Cell proliferation analysis. To measure cell proliferation, cells were seeded at a low density $\left(2 \times 10^{4} /\right.$ well) in 24 -well plates and cultured as described earlier. The number of cells was counted every $24 \mathrm{~h}$ in the following 6 days. A growth curve was constructed according to the data. Three replicates each of three independent experiments were performed.

Wound-healing assay. Cells were grown to confluency and wounded by dragging a $10-\mu 1$ pipette tip through the monolayer. The cells were washed to remove cellular debris and allowed to migrate for $48 \mathrm{~h}$. Images were captured by microscopy (Olympus, Tokyo, Japan) at 0, 24 and $48 \mathrm{~h}$ time-points following wounding. The widths of the wounded areas were measured at $0 \mathrm{~h}\left(\mathrm{~W}_{0}\right)$ and $48 \mathrm{~h}\left(\mathrm{~W}_{48}\right)$ time-points. The relative migration distance (\% of recovery) was calculated as $\left(\mathrm{W}_{0}-\mathrm{W}_{48}\right) / \mathrm{W}_{0} \times 100 \%$. Five replicates each of three independent experiments were performed.

Western blot analysis. Cells were harvested and lysed in

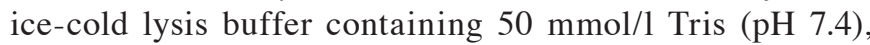
$150 \mathrm{mmol} / 1 \mathrm{NaCl}, 1 \mathrm{mmol} / 1 \mathrm{NaHCO}_{3}, 1 \%$ Triton X-100, $20 \mathrm{mmol} / \mathrm{l} \mathrm{EDTA}, 1 \mathrm{mmol} / \mathrm{l} \mathrm{NaF}, 1 \mu \mathrm{g} / \mathrm{ml}$ leupeptine and $10 \mathrm{mmol} / \mathrm{l}$ phenylmethyl sulfonylfluoride. The protein concentration was determined by BCA protein assay kit (Pierce Biotechnology, Inc., Rockford, IL, USA). Twenty micrograms of each sample were applied to 10\% SDS-polyacrylamide gel. Proteins were transferred onto a nitrocellulose membrane (Millipore) and incubated with each primary antibody, diluted at 1:1,000 (Santa Cruz Biotechnology, Inc., Santa Cruz, CA, USA) overnight at $4^{\circ} \mathrm{C}$, followed by incubation for $1 \mathrm{~h}$ at room temperature with a horseradish peroxidase-conjugated secondary antibody diluted 1:1,000. The blots were then stained using an ECL western blotting system (GE, Fairfield, CT, USA).

Gelatin zymography. The gelatinolytic activity of MMP-2 in the conditioned medium was determined using zymography. Cells were cultured in serum-free DMEM for $24 \mathrm{~h}$ and the supernatants were collected following centrifugation at $500 \mathrm{xg}$ for $10 \mathrm{~min}$. Equal amounts of total protein were loaded onto a zymogram gel composed of $10 \%$ polyacrylamide with $1 \mathrm{mg} / \mathrm{ml}$ gelatin. Samples were electrophoresed at a constant voltage of $130 \mathrm{~V}$ for $2 \mathrm{~h}$. The gel was washed for $40 \mathrm{~min}$ in $2.5 \%$ Triton $\mathrm{X}-100$ to renature the proteins and incubated for $40 \mathrm{~h}$ in $50 \mathrm{mmol} / \mathrm{l}$ Tris- $\mathrm{HCl}(\mathrm{pH} 7.6)$ containing $5 \mathrm{mmol} / \mathrm{l}$ $\mathrm{CaCl}_{2}$ at $37^{\circ} \mathrm{C}$. Following staining with $1 \%$ Coomassie blue and destaining, the proteolytic activity was detected as clear bands on blue background gel. Three independent experiments were performed.

Statistical analysis. Statistical analysis was performed using the SPSS software package (version 13.0.0; SPSS, Inc., Chicago, IL, USA). The Student's unpaired t-test was performed to determine statistically significant differences. $\mathrm{P}<0.05$ was considered to indicate a statistically significant difference.

\section{Results}

Claudin- 6 expression in the HBL-100 cell line is knocked down by shRNA. Claudin- 6 expression was examined by RT-PCR in the breast cancer cell lines MDA-MB-231 and MCF-7 and in the normal human breast epithelial cell line HBL-100. Claudin-6 mRNA expression was detected only in HBL-100 but not in the other two breast cancer cell lines (Fig. 1A).

To investigate whether claudin- 6 plays any role in suppressing the development of breast cancer, shRNA was used to knock down claudin- 6 expression in the HBL-100 cell line. The expression of claudin- 6 mRNA was reduced by $50 \%$ in shRNA-4-expressing cells, which was the most significantly decreased of the four shRNA sequences. Claudin- 6 expression remained unchanged in the cells expressing control shRNA (Fig. 1B). The decreased expression of claudin-6 protein in shRNA-4-expressing cells was confirmed by western blot analysis using anti-claudin-6 antibody (Fig. 1C). 
A

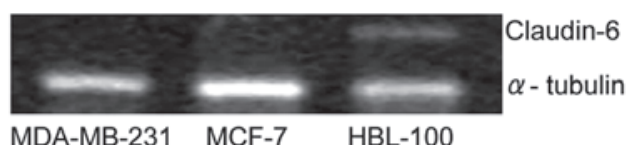

MDA-MB-231 MCF-7 HBL-100

B
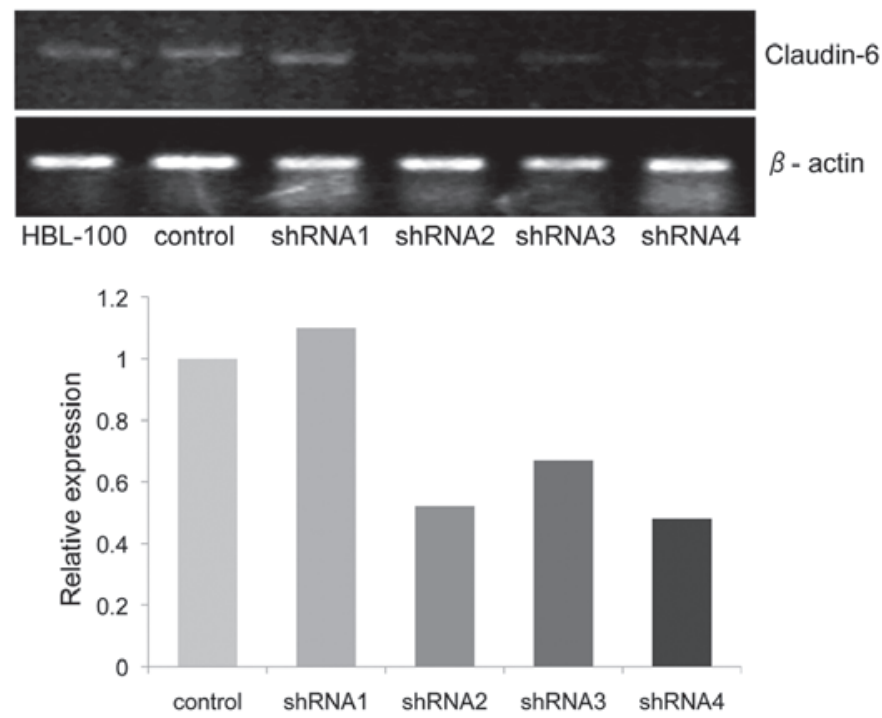

C

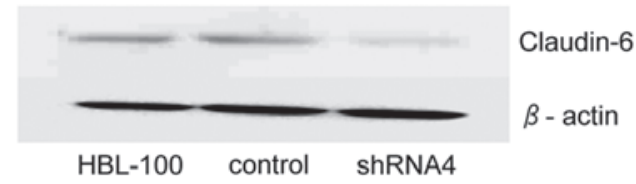

HBL-100 control shRNA4

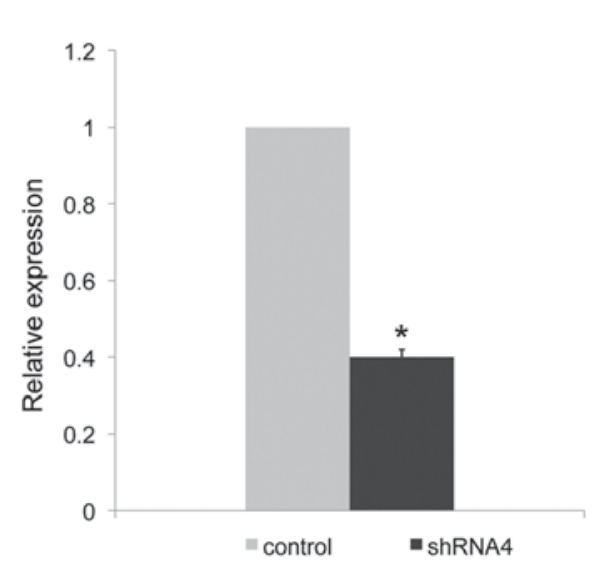

Figure 1. Claudin-6 expression was knocked down by shRNA in the HBL-100 cell line. (A) mRNA expression of claudin-6 in MDA-MB-231, MCF-7 and HBL-100 cell lines was detected by RT-PCR, using $\alpha$-tubulin levels as a loading control. The data shown are representative of three separate experiments. (B) Expression of claudin- 6 mRNA in four shRNA-expressing cells was determined by RT-PCR, compared with HBL-100 cells and negative control shRNA cells, using $\beta$-actin levels as a loading control. The brilliance of each band was used to calculate the relative expression following normalization to the respective $\beta$-actin density. The data shown are representative of three separate experiments. (C) The reduced expression of claudin- 6 protein in shRNA-4-expressing cells was determined by western blot analysis, compared with the HBL-100 and negative control shRNA cells, using $\beta$-actin levels as the loading control. The density of each band was used to calculate the relative expression following normalization to the respective $\beta$-actin density. The data shown are representative of three separate experiments. shRNA, short hairpin RNA; RT-PCR, reverse transcription-polymerase chain reaction.

TER is decreased in claudin-6 knockdown cells. Claudin expression is known to be important for tight junction formation. TER across confluent epithelial cell layers is a measurement of tight junction function. When cells reached confluency, we measured TER to evaluate whether knockdown of claudin-6 in HBL-100 cells may result in any changes in the function of tight junctions. Compared with control cells, the TER of claudin- 6 knockdown cells was decreased and the differences were found to be statistically significant $(\mathrm{P}<0.001$; Fig. 2A). The results indicate that the function of tight junction is changed by knockdown of claudin- 6 in HBL-100 cells.

Claudin-6 knockdown cells have a higher proliferation rate and migratory ability. To determine the effect of claudin- 6 knockdown on the proliferation of HBL-100 cells, growth curves were constructed over a period of 6 days by manual cell counting. The proliferation rate of claudin- 6 knockdown cells was significantly higher than that in the control cells $(\mathrm{P}<0.001$; Fig. 2B). The cell migratory ability was compared between the claudin- 6 knockdown cells and control cells, using a wound healing assay. Following 24 and 48 h wound generation in the monolayers of cells, the wound of the control cells was wider than that of the claudin- 6 knockdown cells. Claudin- 6 interference resulted in a $100 \%$ increase in the migration rate $(\mathrm{P}<0.001$; Fig. 2C).
Claudin-6 knockdown cells have higher expression and activity of MMP-2. As expression of MMP-2 is associated with cell migration, changes in MMP-2 expression and activity were examined by RT-PCR and gelatin zymography assay to investigate associations with the migratory phenotype. The MMP-2 mRNA expression of the claudin- 6 knockdown cells was 2.6-fold higher than that of the control cells $(\mathrm{P}<0.001$; Fig. 3A). While the zymography assay result revealed that the MMP-2 activity of claudin-6 knockdown cells was 3-fold higher than that of the control cells $(\mathrm{P}<0.001$; Fig. 3B). The increase in MMP-2 activity caused by claudin- 6 knockdown was associated with the results of cell migration, indicating that claudin-6 knockdown may increase the cell migration ability through activation of MMP-2.

Claudin-6 knockdown cells have lower phosphorylated p38 MAPK expression. It has been reported that the p38 MAPK signaling pathway was involved in cell proliferation and MMP activation in other cell lines (12-14). To test whether claudin-6 knockdown had any effect on the p38 MAPK pathway, the level of p38 MAPK and phosphorylated p38 MAPK protein expression was investigated by western blot analysis. Results showed a $41 \%$ decrease in phosphorylated p38 MAPK expression of claudin- 6 knockdown cells $(\mathrm{P}<0.001)$, while the $\mathrm{p} 38$ MAPK expression remained unchanged (Fig. 4). The present 
A

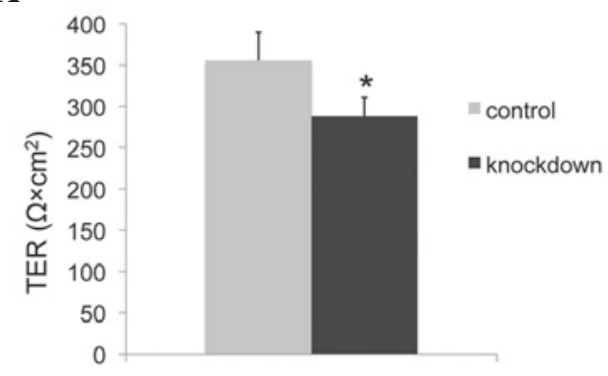

B

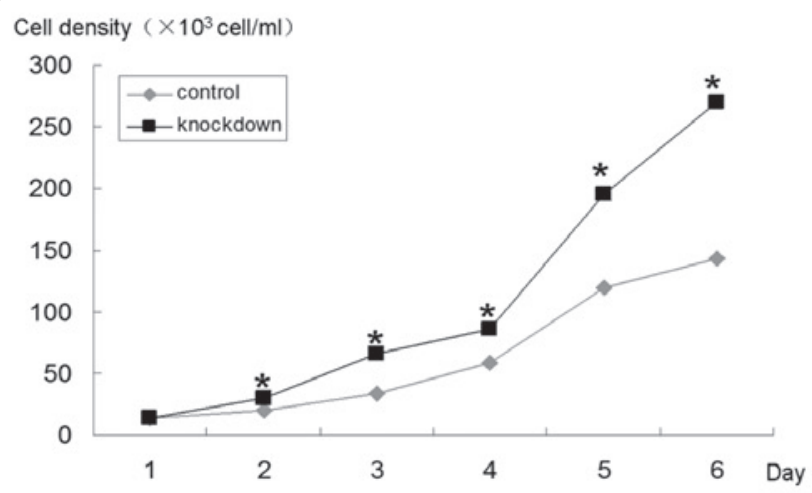

C
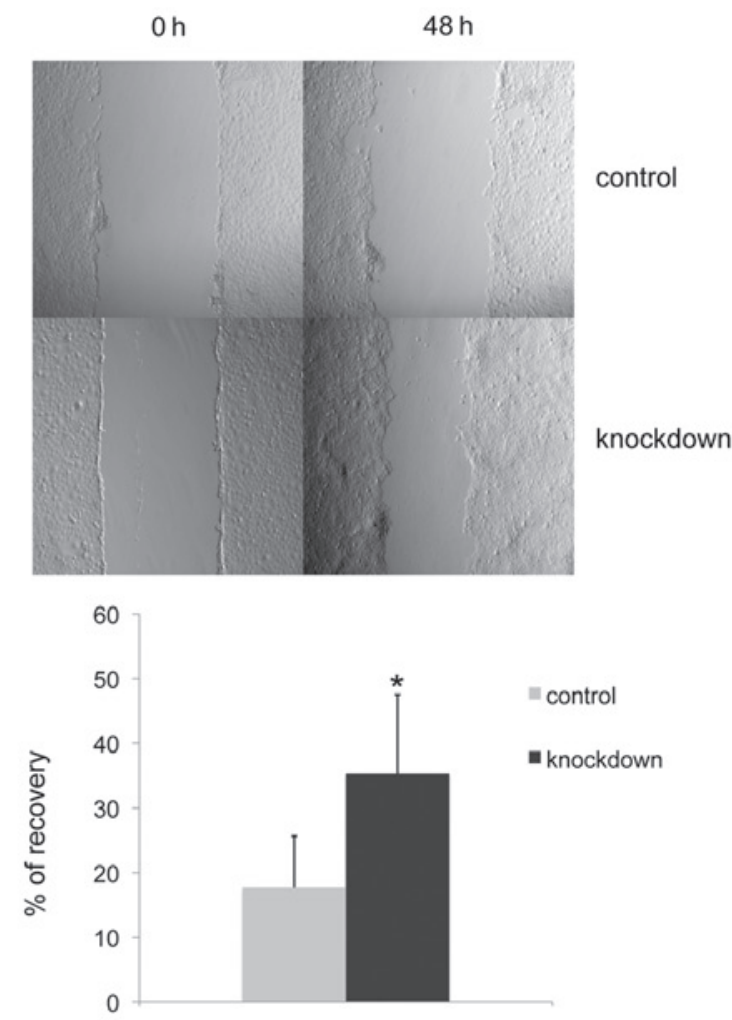

Figure 2. Effect of claudin-6 knockdown on TER, cell proliferation and migration. (A) The TER of claudin-6 knockdown cells was significantly lower than that of cells in the control group. TER was measured by Millicell-ERS volt- $\Omega$ meter. Data are presented as mean \pm SEM $(n=3)$ and the data shown are representative of three separate experiments. "P<0.001 vs. negative control shRNA group. (B) The proliferation of claudin- 6 knockdown cells was significantly higher compared with the control cells. A growth curve was constructed from data obtained by manual cell counting over 6 days. Data are presented as mean \pm SEM $(\mathrm{n}=3)$ and the data shown are representative of three separate experiments. ${ }^{*} \mathrm{P}<0.001$ vs. negative control shRNA group. (C) Wound healing assay showed cell migration of claudin-6 knockdown cells was higher compared with the control group. The width of the wounded area at $0 \mathrm{~h}\left(\mathrm{~W}_{0}\right)$ and $48 \mathrm{~h}\left(\mathrm{~W}_{48}\right)$ were measured. The relative migration distance (\% of recovery) was calculated as $\left(\mathrm{W}_{0}-\mathrm{W}_{48}\right) / \mathrm{W}_{0} \times 100 \%$. Data are presented as mean $\pm \mathrm{SEM}(\mathrm{n}=5)$ and the data shown are representative of three separate experiments. ${ }^{~} \mathrm{P}<0.001$ vs. negative control shRNA group. TER, transepithelial electrical resistance; shRNA, short hairpin RNA.

A
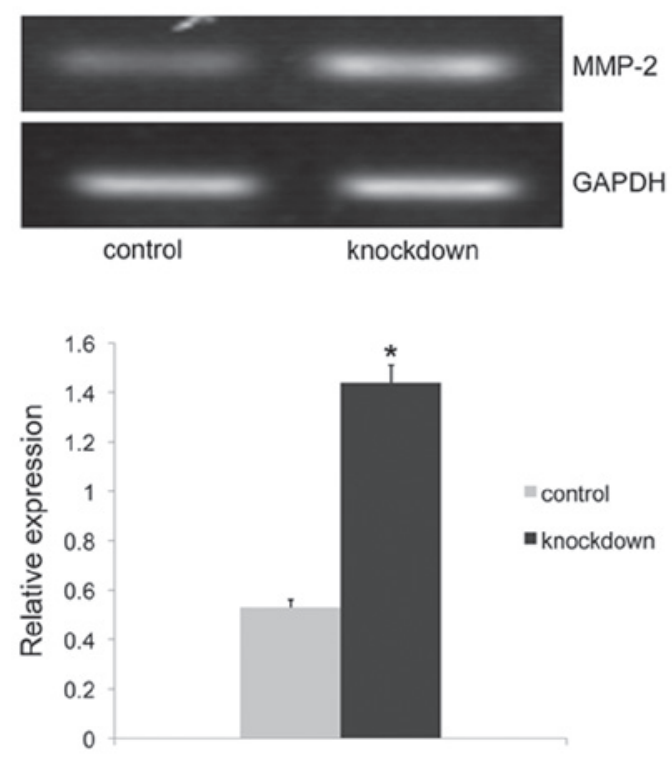

B
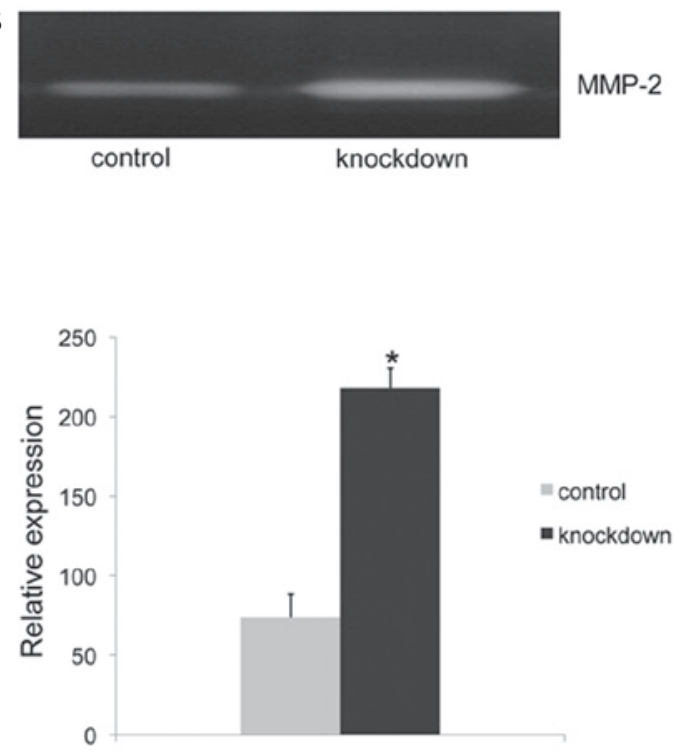

Figure 3. Effect of claudin-6 knockdown on MMP-2 expression and activity. (A) The upregulated expression of MMP-2 was observed by RT-PCR in claudin-6 knockdown cells, compared with the HBL-100 and negative control shRNA cells. GAPDH levels were used as a loading control. The density of each band was used to calculate the relative expression following normalization to the respective GAPDH density. The data shown are representative of three separate experiments. "P<0.001 vs. negative control shRNA group. (B) The MMP-2 activity of claudin- 6 knockdown cells was significantly higher compared with the control cells, as detected by a gelatin zymography assay. The density of each band was used to calculate the relative expression levels. The data shown are representative of three separate experiments. ${ }^{*} \mathrm{P}<0.001$ vs. negative control shRNA group. MMP-2, matrix metalloproteinase-2; RT-PCR, reverse transcription-polymerase chain reaction; shRNA, short hairpin RNA. 

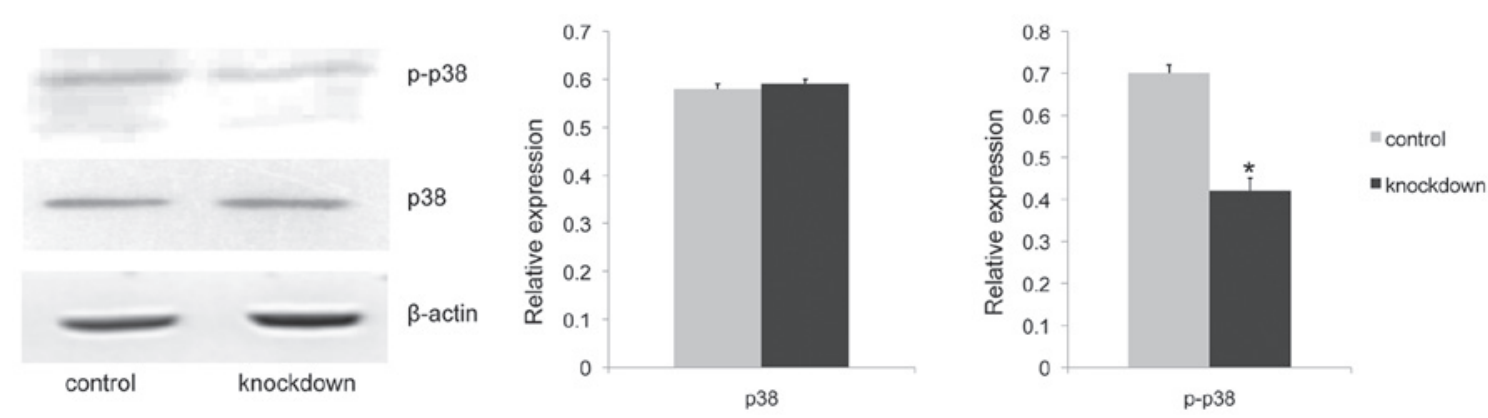

Figure 4. Effect of claudin-6 knockdown on the p38 MAPK pathway. Expression of p38 and p-p38 protein was determined by western blot analysis, the p-p38 expression in claudin- 6 knockdown cell decreased while the $\mathrm{p} 38$ expression was unchanged, compared with the HBL-100 and negative control shRNA cells, $\beta$-actin levels were used as a loading control. The density of each band was used to calculate the relative expression following normalization to respective $\beta$-actin densities. The data shown are representative of three separate experiments. "P $<0.001$ vs. negative control shRNA group. MAPK, mitogen-activated protein kinase; p-p38, phosphorylated p38; shRNA, short hairpin RNA.

results confirmed the correlation between claudin- 6 expression and p38 MAPK pathway activation.

\section{Discussion}

One of the first steps in cancer metastasis is loss of cell-to-cell adhesion $(15,16)$. Claudins are known to be important for tight junction formation and the changes in their expression or localization often leads to the disruption or deregulation of the tight junction structure and function, which play a critical role in cancer progression, invasion and metastasis. TER across the confluent epithelial cell layer is a measurement of tight junction function. Overexpression of various claudins, including claudin- 1 and -4 , leads to increased TER $(17,18)$, while the downregulation of various types of claudins may lead to decreased TER and altered tight junction structures, including claudin-3, -4 (19), -5 (20) and -7 (21). Overexpression of claudin- 6 was observed to lead to an increased TER in the MCF-7 breast cancer cell line (11). Consistent with this previous observation, the current study found that the TER of claudin-6 knockdown cells was lower than the control cells, indicating that the organization or function of tight junction is disrupted by the downregulation of claudin- 6 in the HBL-100 cell line.

In the current study, significantly higher rates of proliferation in claudin-6 knockdown cells, compared with control cells were observed. In addition, the downregulated expression of claudin- 6 was found to lead to an increased migratory ability in HBL-100 cells with a higher mRNA expression and activity of MMP-2, suggesting that the cells gained a relatively, more malignant phenotype following claudin- 6 knockdown mediated via the activation of MMP-2. Furthermore, a decreased expression of phosphorylated p38 MAPK in claudin- 6 knockdown cells was also found in the current study, indicating that p38 MAPK may be one of the signaling pathways involved in altering the phenotype of the cells following claudin- 6 knockdown. To the best of our knowledge, this is the first report in which silencing of the claudin- 6 gene enhanced cell proliferation and migration, accompanied with MMP-2 activation via the p38 MAPK pathway in HBL-100 cells.

Cell proliferation and migration are essential for cancer progression, invasion and metastasis. Evidence has shown the effect of claudin on cell proliferation; findings of a previous study showed that the overexpression of claudin- 6 resulted in a lower proliferation rate of MCF-7 cells (11) and it has been reported by $\mathrm{Li}$ et al (22) that the overexpression of miR-7 and miR-218 was accompanied by reactivation of claudin- 6 gene, leading to the inhibition of the cell cycle and clone formation of breast cancer cells, which markedly supports the results of the current hypothesis. Sun et al (23) have also reported that the downregulated expression of claudin-3 reduces cell proliferation. The mechanism by which claudins affect proliferation is complex and involves a number of signaling pathways, including p38 MAPK.

It has been reported that the p38 MAPK signaling pathway negatively regulates cell proliferation in other cell lines. The mechanism by which p38 MAPK regulates cell proliferation remains unclear, however there are a number of studies suggesting the involvement of the JNK/c-Jun pathway $(24,25)$, EGFR (26) and p53 (27). p38 also is involved in p21 upregulation, subsequently resulting in cell growth inhibition (28). Increased proliferation of hepatocytes has been observed during liver regeneration in mice lacking p38 $\alpha$ in the liver (12).

As well as the changed proliferation ability, higher migration ability in claudin-6 knockdown cells was observed. Cell migration requires MMPs, serine proteases and a number of other proteases. Numerous types of claudin have been shown to change cancer metastasis by the regulation of MMP activity. For example, overexpression of claudin- 4 increased MMP-2 and -9 activity in Caco-2 cells (29) and knockdown of claudin-2 increased cell migration and MMP-9 activity in MDCK cells (30). These studies suggest the potential involvement of claudins in cancer metastasis via MMP activation. Consistent with the current observations, Osanai et al (31) demonstrated that knockdown of claudin- 6 resulted in increased MMP activity in MCF-7 cells. Although claudins have been shown to activate MMPs $(8,32)$, the mechanism of the enhancement of MMP-2 activity by claudin- 6 knockdown is not yet understood. In the current study, a decreased expression of phosphorylated p38 MAPK following claudin-6 knockdown was observed. p38 MAPK has been shown to be involved in MMP-2 and -9 regulation. Green et al have reported that inhibition of the $\mathrm{p} 38$ MAPK pathway resulted in a $228 \%$ increase in MMP-2 secretion (13) and Kim et al demonstrated that TGF- $\beta$ induced the upregulation of MMP- 2 and -9 through p38 MAPK signaling in the human breast epithelial 
cell line MCF10A (14), providing increasing evidence of the correlation between MMP-2 activation and the p38 MAPK pathway. Since there is little understanding of the mechanism by which p38 MAPK pathway is involved in MMP-2 activity, the potential transcription factors involved in it are now under investigation. However, further studies are required to address the exact mechanism by which claudin- 6 function is regulated through the p38 MAPK signaling pathway to alter the phenotype of cells.

In conclusion, the current study has demonstrated that downregulation of claudin- 6 expression leads to a relatively malignant phenotype mediated through p38 MAPK signaling pathway in the human breast epithelium cell line HBL-100, further supporting the hypothesis that claudin- 6 is likely to be a suppressor in a number of types of cancer. Decreased expression of claudin- 6 may contribute to the malignant progression of breast cancer.

\section{Acknowledgements}

This study was supported by grants from the National Natural Science Foundation of China (no. 81172499) and Science and Technology Development Plan of the Office of Science and Technology Project in Jilin Province (no. 201115113). The authors would like to thank William Orr, Department of Pathology, University of Manitoba, Canada, for providing language help with this manuscript.

\section{References}

1. Elkouby-Naor L and Ben-Yosef T: Functions of claudin tight junction proteins and their complex interactions in various physiological systems. Int Rev Cell Mol Biol 279: 1-32, 2010.

2. Tsukita S, Furuse M and Itoh M: Multifunctional strands in tight junctions. Nat Rev Mol Cell Biol 2: 285-293, 2001.

3. Matter K, Aijaz S, Tsapara A and Balda MS: Mammalian tight junctions in the regulation of epithelial differentiation and proliferation. Curr Opin Cell Biol 17: 453-458, 2005.

4. González-Mariscal L, Betanzos A, Nava P and Jaramillo BE: Tight junction proteins. Prog Biophys Mol Biol 81: 1-44, 2003.

5. Takahashi A, Kondoh M, Kodaka M and Yagi K: Peptides as tight junction modulators. Curr Pharm Des 17: 2699-2703, 2011.

6. Lee KW, Lee NK, Kim JH, et al: Twist1 causes the transcriptional repression of claudin- 4 with prognostic significance in esophageal cancer. Biochem Biophys Res Commun 423 . 454-460, 2012.

7. Tokés AM, Kulka J, Paku S, et al: Claudin-1, -3, and -4 proteins and mRNA expression in benign and malignant breast lesions: a research study. Breast Cancer Res 7: R296-R305, 2005.

8. Miyamori H, Takino T, Kobayashi Y, Tokai H, Itoh Y, Seiki M and Sato $\mathrm{H}$ : Claudin promotes activation of pro-matrix metalloproteinase- 2 mediated by membrane-type matrix metalloproteinases. J Biol Chem 276: 28204-28211, 2001.

9. Turksen K and Troy TC: Junctions gone bad: claudins and loss of the barrier in cancer. Biochim Biophys Acta 1816: 73-79, 2011.

10. Quan C and Lu SJ: Identification of genes preferentially expressed in mammary epithelial cells of Copenhagen rat using subtractive hybridization and microarrays. Carcinogenesis 24: 1593-1599, 2003.

11. Wu Q, Liu Y, Ren Y, Xu X, Yu L, Li Y and Quan C: Tight junction protein, claudin- 6 , downregulates the malignant phenotype of breast carcinoma. Eur J Cancer Prev 19: 186-194, 2010.

12. Hui L, Bakiri L, Stepniak E and Wagner EF: p38alpha: a suppressor of cell proliferation and tumorigenesis. Cell Cycle 6: 2429-2433, 2007.
13. Green JA, Dholakia S, Friedland JS, et al: Mycobacterium tuberculosis-infected human monocytes down-regulate microglial MMP-2 secretion in CNS tuberculosis via TNF $\alpha, N_{\kappa} \mathrm{B}, \mathrm{p} 38$ and caspase 8 dependent pathways. J Neuroinflammation 8: 46, 2011.

14. Kim ES, Kim MS and Moon A: TGF-beta-induced upregulation of MMP-2 and MMP-9 depends on p38 MAPK, but not ERK signaling in MCF10A human breast epithelial cells. Int J Oncol 25: 1375-1382, 2004

15. Hazan RB, Qiao R, Keren R, Badano I and Suyama K: Cadherin switch in tumor progression. Ann NY Acad Sci 1014: 155-163, 2004.

16. Jordan NV, Johnson GL and Abell AN: Tracking the intermediate stages of epithelial-mesenchymal transition in epithelial stem cells and cancer. Cell Cycle 10: 2865-2873, 2011.

17. Bhat AA, Sharma A, Pope J, Krishnan M, Washington MK, Singh $\mathrm{AB}$ and Dhawan P: Caudal homeobox protein $\mathrm{cdx}-2$ cooperates with Wnt pathway to regulate claudin-1 expression in colon cancer cells. PLoS One 7: e37174, 2012.

18. Neesse A, Griesmann H, Gress TM and Michl P: Claudin-4 as therapeutic target in cancer. Arch Biochem Biophys 524: 64-70, 2012.

19. Lambert D, Padfield PJ, McLaughlin J, Cannell S and O'Neill CA: Ochratoxin A displaces claudins from detergent resistant membrane microdomains. Biochem Biophys Res Commun 358: 632-636, 2007.

20. Haorah J, Heilman D, Persidsky Y, et al: Ethanol-induced activation of myosin light chain kinase leads to dysfunction of tight junctions and blood-brain barrier compromise. Alcohol Clin Exp Res 29: 999-1009, 2005.

21. Oshima T, Miwa H and Joh T: Aspirin induces gastric epithelial barrier dysfunction by activating p38 MAPK via claudin-7. Am J Physiol Cell Physiol 295: C800-C806, 2008.

22. Li Q, Zhu F and Chen P: miR-7 and miR-218 epigenetically control tumor suppressor genes RASSF1A and Claudin-6 by targeting HoxB3 in breast cancer. Biochem Biophys Res Commun 424: 28-33, 2012.

23. Sun C, Yi T, Song X, et al: Efficient inhibition of ovarian cancer by short hairpin RNA targeting claudin-3. Oncol Rep 26: 193-200, 2011.

24. Hui L, Bakiri L, Mairhorfer A, et al: p38alpha suppresses normal and cancer cell proliferation by antagonizing the JNK-c-Jun pathway. Nat Genet 39: 741-749, 2007.

25. Perdiguero E, Ruiz-Bonilla V, Gresh L, et al: Genetic analysis of p38 MAP kinases in myogenesis: fundamental role of p38alpha in abrogating myoblast proliferation. EMBO J 26: 1245-1256, 2007.

26. Ventura JJ, Tenbaum S, Perdiguero E, Huth M, Guerra C, Barbacid M, Pasparakis $M$ and Nebreda AR: p38alpha MAP kinase is essential in lung stem and progenitor cell proliferation and differentiation. Nat Genet 39: 750-758, 2007.

27. Bulavin DV and Fornace AJ Jr: p38 MAP kinase's emerging role as a tumor suppressor. Adv Cancer Res 92: 95-118, 2004.

28. Lee B, Kim CH and Moon SK: Honokiol causes the p21WAF1-mediated G(1)-phase arrest of the cell cycle through inducing p38 mitogen activated protein kinase in vascular smooth muscle cells. FEBS Lett 580: 5177-5184, 2006.

29. Takehara M, Nishimura T, Mima S, Hoshino T and Mizushima T: Effect of claudin expression on paracellular permeability, migration and invasion of colonic cancer cells. Biol Pharm Bull 32: 825-831, 2009.

30. Ikari A, Takiguchi A, Atomi K, Sato T and Sugatani J: Decrease in claudin-2 expression enhances cell migration in renal epithelial Madin-Darby canine kidney cells. J Cell Physiol 226: 1471-1478, 2011.

31. Osanai M, Murata M, Chiba H, Kojima T and Sawada N: Epigenetic silencing of claudin-6 promotes anchorage-independent growth of breast carcinoma cells. Cancer Sci 98 : 1557-1562, 2007.

32. Agarwal R, D'Souza T and Morin PJ: Claudin-3 and claudin-4 expression in ovarian epithelial cells enhances invasion and is associated with increased matrix metalloproteinase- 2 activity. Cancer Res 65: 7378-7385, 2005. 